\title{
Vom Studium in den Alltag als Arzt und Ärztin in Weiterbildung
}

\author{
Larissa Luchsinger $^{a}$, Anne Berthold ${ }^{b}$, Werner Bauer $^{c}$, Monika Brodmann Maeder ${ }^{\text {, Michael Siegrist }}$ \\ a MA, wissenschaftliche Mitarbeiterin, Consumer Behavior, ETH Zürich; b Dr., Senior Researcher, Consumer Behavior, ETH Zürich; \\ ${ }^{c}$ Dr. med., ehemaliger Präsident SIWF; d PD Dr. med., MME, Präsidentin SIWF; e Prof. Dr. phil., Professor für Consumer Behavior, ETH Zürich
}

\begin{abstract}
Der Einstieg in den Berufsalltag stellt junge Ärztinnen und Ärzte in Weiterbildung vor grosse Herausforderungen. Die jährlich durchgeführte Befragung selektiert auch wechselnde Themen, die immer wieder oder speziell in der untersuchten Periode aktuell sind. 2003 und 2008 erfolgte eine Befragung zum Thema Qualität der medizinischen Ausbildung in Bezug auf die Vorbereitung für die ärztliche Weiterbildung. Dieses Thema wurde in der Befragung 2019 erneut aufgegriffen.
\end{abstract}

Zum Zeitpunkt des Studienabschlusses der 2019 befragten Ärztinnen und Ärzte boten fünf medizinische Fakultäten in der Schweiz - Genf, Lausanne, Zürich, Basel und Bern - ein komplettes Studium der Humanmedizin an. ${ }^{1}$ Die Modulfragen beziehen sich in der Befragung 2019 auf diese ursprünglichen fünf Fakultäten. Im Jahr 2017 wurde gesamtschweizerisch der bisherige Lernzielkatalog "Swiss Catalogue of Learning Objectives for Undergraduate Medical Training SCLO» durch «PROFILES» (Principal Relevant Objectives and a Framework for Integrative Learning and Education in Switzerland) ersetzt $[1,2]$. Die Umsetzung des damit verbundenen kompetenzorientierten Ansatzes, der unter anderem kommunikative Aspekte, Professionalität, Interprofessionalität und die Fähigkeit, einen kritischen Blick auf den Fortschritt in der Medizin zu entwickeln, bein-

Das Medizinstudium soll den angehenden Ärztinnen und Ärzten selbständiges Arbeiten im Berufsalltag ermöglichen.

1 In der Zwischenzeit sind Fakultäten dazugekommen, die einen Bacheloroder Masterab-

schluss (ETH Zürich bzw. Università della Svizzera Italiana) oder einen kompletten Studiengang anbieten (Universität Freiburg i.Üe.). haltet, ist an den medizinischen Fakultäten unterschiedlich weit fortgeschritten. Als übergeordnetes Ziel soll das Medizinstudium die angehenden Ärztinnen und Ärzte möglichst gut auf ihre Tätigkeit vorbereiten und ihnen einen guten Einstieg und selbständiges Arbeiten im Berufsalltag ermöglichen. Das Ziel der Modulfragen 2019 war es zu erfassen, wie gut dieses Ziel erfüllt wurde und ob sich in der aktuellen Untersuchung im Vergleich zu den früheren Erhebungen Unterschiede zeigen.

\section{Methode und Fragebogen}

Für die Umfrage von 2019 erhielten alle 12088 gemeldeten Ärztinnen und Ärzte in Weiterbildung einen

Die Teilnehmenden wurden auch gefragt, inwieweit sie durch das Studium gelernt hätten, soziale Faktoren bei einer Entscheidung zu berücksichtigen.

Fragebogen, wovon 8380 diesen retournierten (Rücklaufquote: 69,3\%). Im Rahmen der Erhebung der statistischen Grundlagen 2019 nahmen zudem 1534 Leiterinnen und Leiter einer Weiterbildungsstätte an der Befragung teil (Rücklaufquote: 95\%). Die Vorgehensweise sowie die Rücklaufquote entsprachen denjenigen der letzten Jahre [3, 4].

Im Modul «Vom Studium in den Alltag als Arzt/Ärztin in Weiterbildung» wurden die Teilnehmenden gebeten einzuschätzen, wie gut sie sich nach dem Studium auf den Berufsalltag vorbereitet fühlten. Zudem wurde erfragt, ob das Studium zu theorielastig gewesen sei und inwieweit sie durch das Studium gelernt hätten, soziale Faktoren bei einer Entscheidung zu berücksichtigen. Die Beantwortung der Fragen erfolgte jeweils auf einer Skala von 1 bis 6 und wurden für eine übersichtlichere Darstellung in drei Kategorien zusammengefasst: trifft (überhaupt) nicht zu (1-2), unentschieden (3-4) und trifft (voll und ganz) zu (5-6). Die Leiterinnen und Leiter der Weiterbildungsstätten konnten in einem separaten Teil zusätzlich beurteilen, wie selbständig die Ärzte und Ärztinnen in Weiterbildung im ers- 


\section{Wie selbstständig können die Ärzte in Weiterbildung im ersten halben Jahr nach ihrem Studium ihre Arbeit erledigen?}

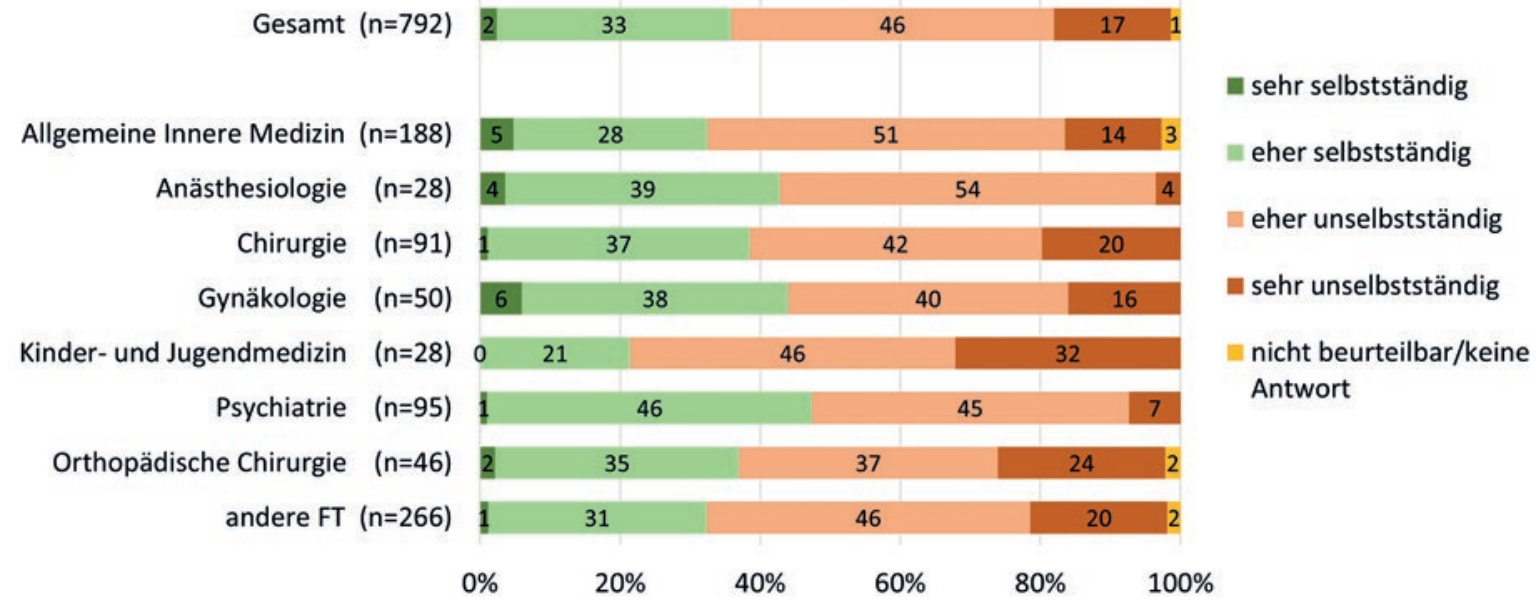

Abbildung 1: Einschätzung der Leiterinnen und Leiter der Weiterbildungsstätten, wie selbständig die Ärztinnen und Ärzte in Weiterbildung im ersten halben Jahr nach dem Studium ihre Arbeit erledigen können, aufgeteilt nach Fachrichtung der Weiterbildungsstätte.

ten halben Jahr nach dem Studium die Arbeit erledigt hatten.

Die Fragen zur universitären Ausbildung wurden bereits in den Jahren 2003 und 2008 den Ärzten und Ärztinnen in Weiterbildung gestellt. Dies ermöglicht einen interessanten Langzeitvergleich.

\section{Fachrichtungen: unterschiedlich selbständig}

Von den Leiterinnen und Leitern der Weiterbildungsstätten sind rund ein Drittel der Meinung, dass die Ärztin- nen und Ärzte in Weiterbildung im ersten halben Jahr nach dem Studium die Arbeit sehr selbständig (2\%) oder eher selbständig erledigen können (33\%). Die anderen zwei Drittel beurteilten ihre Ärztinnen und Ärzte in Weiterbildung als eher unselbständig (46\%) oder stuften sie als sehr unselbständig ein (17\%). Werden die Fachrichtungen separat betrachtet, zeigt sich, dass die Ärztinnen und Ärzte in Weiterbildung in der Kinder- und Jugendmedizin als am wenigsten selbständig beurteilt werden. Als am selbständigsten werden die Ärztinnen und Ärzte in Weiterbildung in den Fachrichtungen Psychiatrie, Gynäkologie und Anästhesiologie wahrgenommen.

Im Studium lernt man, bei einer Entscheidung auch die sozialen Faktoren zu berücksichtigen.

Ich fühlte mich nach meinem Studium gut auf den Berufsalltag als Arzt/Ärztin in Weiterbildung vorbereitet.

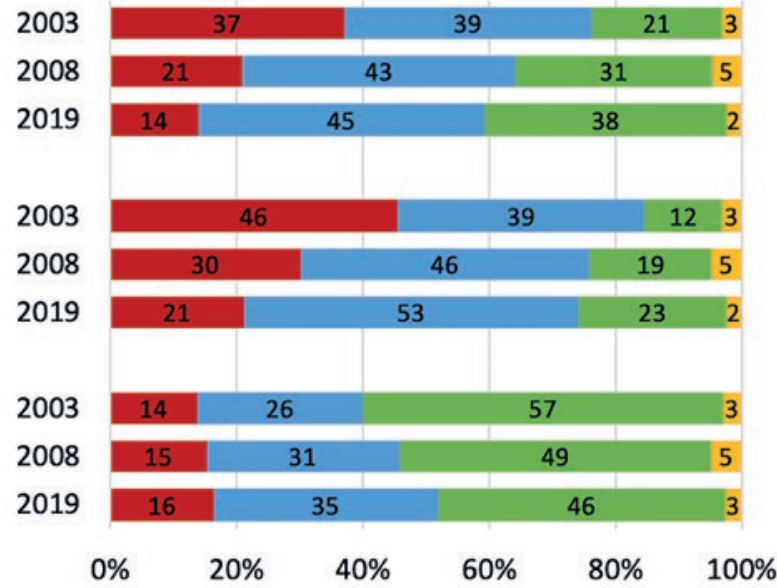

- trifft (überhaupt) nicht zu $\quad$ unentschieden

w trifft (voll und ganz) zu $\quad$ keine Angaben

Abbildung 2: Prozentuale Antworten der drei Items zur universitären Ausbildung, beurteilt auf einer Skala von 1 (trifft gar nicht zu) bis 6 (trifft voll und ganz zu) für die Jahre 2003 ( $n=5343$ ), 2008 ( $n=6011)$ und 2019 ( $n=8380$ ).

Für die Grafik wurden die sechs Antwortkategorien in drei Kategorien zusammengefasst. 


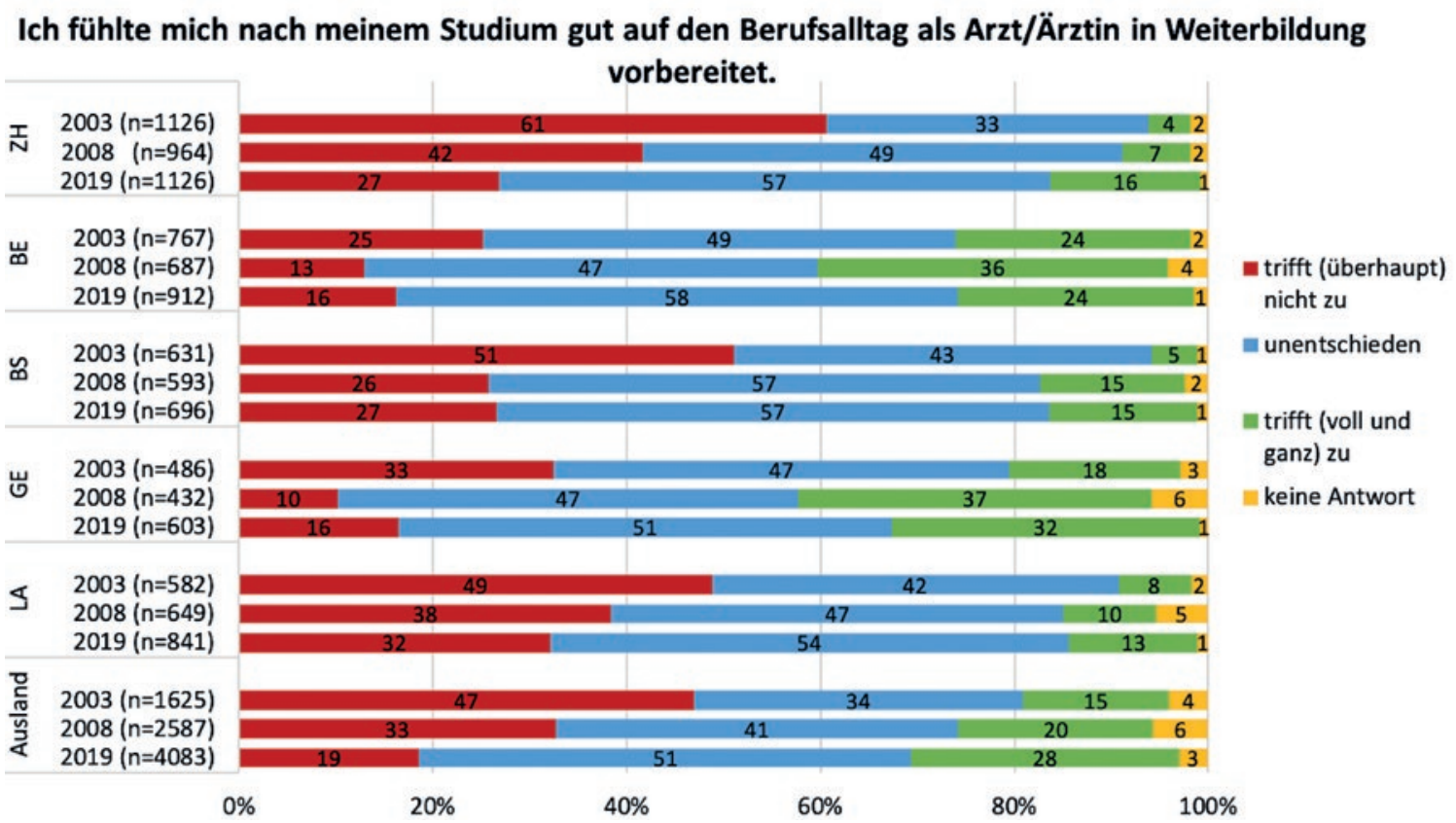

Abbildung 3: Prozentuale Antworten zur Aussage «lch fühlte mich nach meinem Studium gut auf den Berufsalltag als Arzt/Ärztin in Weiterbildung vorbereitet", beurteilt auf einer Skala von 1 (trifft gar nicht zu) bis 6 (trifft voll und ganz zu). Für die Grafik wurden die sechs Antwortkategorien in drei Kategorien zusammengefasst.

\section{Universitäre Ausbildung wurde praxisnaher}

Im Jahr 2019 fühlten sich 23\% der Ärztinnen und Ärzte in Weiterbildung nach dem Studium sehr gut auf den Berufsalltag vorbereitet und über die Hälfte (53\%) als teilweise gut. 2003 war dies nur bei 12\% resp. 39\% und 2008 bei 19\% resp. 46\% der Fall. Die Absolventen und Absolventinnen fühlen sich heute somit deutlich besser auf den Beruf vorbereitet.

Neben der Vorbereitung auf den Berufsalltag wurden auch die sozialen Faktoren im Studium verstärkt behandelt. 2019 stimmten 38\% der Ärztinnen und Ärzte

Die Resultate zeigen, dass die Ärztinnen und Ärzte in Weiterbildung das Medizinstudium im Vergleich zu früher als praxisnaher wahrnehmen.

in Weiterbildung der Aussage zu, dass bei einer Entscheidung auch gelernt werde, vermehrt soziale Faktoren zu berücksichtigen. Fast die Hälfte (45\%) bejaht diese Aussage zumindest teilweise. 2003 stimmten vergleichsweise weniger (21\% resp. 39\%) dieser Aussage vollkommen resp. teilweise zu.

Von $46 \%$ der Ärztinnen und Ärzte in Weiterbildung wird aber auch kritisiert, dass das Studium immer noch zu theorielastig sei und dass praktische Fragen zu wenig behandelt würden. Im Langzeitvergleich kann dennoch auch bei diesem Item eine leichte Verbesse- rung festgestellt werden; so waren es 2003 57\% und 2008 49\% der Ärztinnen und Ärzte in Weiterbildung, welche dieser Aussage zustimmten. Der Langzeitvergleich zeigt, dass die universitäre Ausbildung und deren Vorbereitung auf den Beruf über die Jahre hinweg immer besser beurteilt und als praxisnaher empfunden wurden.

Wie gut das Studium auf den Berufsalltag vorbereitet, wird je nach Universität, an welcher die Ärztinnen und Ärzte in Weiterbildung ihren Abschluss gemacht haben, unterschiedlich beurteilt. Von den fünf medizinischen Fakultäten schneiden Bern und Genf am besten ab. Bei der Befragung 2019 fühlen sich von Genf $32 \%$ und von Bern $24 \%$ der Ärztinnen und Ärzte in Weiterbildung gut oder sehr gut durch ihr Studium auf ihren Berufsalltag vorbereitet. Mehr als die Hälfte (51\% resp. 58\%) stimmen der Aussage teilweise zu. Bei den anderen drei Universitäten Zürich, Basel und Lausanne fühlen sich zwischen 13\% und 15\% der Ärztinnen und Ärzte in Weiterbildung sehr gut durch ihr Studium auf den Berufsalltag vorbereitet, und 54-57\% stimmen der Aussage teilweise zu. Ärztinnen und Ärzte in Weiterbildung, welche ihren Abschluss ausserhalb der Schweiz erworben haben, fühlen sich zu etwas mehr als einem Viertel gut oder sehr gut vorbereitet (28\%). Im Langzeitvergleich können bei allen fünf Universitäten deutliche Verbesserungen in der Beurteilung festgestellt werden. Ausserdem zeigen sich 2019 geringere Unterschiede zwischen den Universitäten. Bei allen 
Universitäten wird die universitäre Ausbildung heute als bessere Vorbereitung auf den Berufsalltag empfunden als früher.

\section{Direkter Austausch der Beteiligten zentral}

Die vorliegenden Resultate zeigen, dass die Ärztinnen und Ärzte in Weiterbildung das Medizinstudium im Vergleich zu früher an allen Fakultäten als praxisnaher wahrnehmen. Neben dem vermehrten Behandeln von praktischen Fragen wird auch der Einbezug von sozialen Faktoren bei der Entscheidungsfindung zunehmend vermittelt. Vor allem die Universitäten, die in früheren Jahren unterdurchschnittlich beurteilt wurden, haben stark aufgeholt. Trotzdem zeigen sich weiterhin Unterschiede innerhalb der Fakultäten. So fühlten sich die Absolventen und Absolventinnen der Universitäten Genf und Bern am besten auf ihren Berufsalltag vorbereitet.

Laut den offiziellen Beschreibungen der Masterstudiengänge an den fünf Universitäten wird beispielsweise in Genf und Bern eng mit verschiedenen Spitälern zusammengearbeitet, und neben dem Wahlstudienjahr, welches etwa 7-10 Monate dauert, werden auch klinische Blockpraktika durchgeführt. Zusätzlich zum üblichen Wahlstudienjahr haben die Studierenden also die Möglichkeit, in einem Spital oder einer Arztpraxis ein kurzes Praktikum zu absolvieren. Die Unterschiede in der Beurteilung der medizinischen Fakultäten könnten also auf diese zusätzliche Möglichkeit der Anwendung praktischer Fähigkeiten zurückzuführen sein. Die genauen Gründe für diese Unterschiede waren allerdings nicht Gegenstand der Befragung und können deshalb nur vermutet werden.

Auch die Leiterinnen und Leiter der Weiterbildungsstätten wurden gebeten einzuschätzen, wie selbständig die Ärzte und Ärztinnen in Weiterbildung ihre Arbeit im ersten halben Jahr nach Abschluss des Masterstudiums erledigen können. Es erstaunt wenig, dass die meisten Leiterinnen und Leiter der Weiterbildungsstätten ihre Ärztinnen und Ärzte in Weiterbildung als noch nicht sehr selbständig beurteilten. Allerdings zeigen sich grosse Unterschiede in den verschiedenen Fachrichtungen: Als am wenigsten selbständig wurden die Weiterzubildenden in der Fachrichtung Kinderund Jugendmedizin beurteilt, während sie in Gynäkologie, Anästhesiologie und Psychiatrie als am selbständigsten wahrgenommen wurden. Insgesamt stuften rund ein Drittel der Leiterinnen und Leiter der Weiterbildungsstätten ihre Ärztinnen und Ärzte in Weiterbildung nach ihrem Studium als (eher) selbständig ein.

Rund ein Drittel der Leiterinnen und Leiter der Weiterbildungsstätten stuften ihre Ärztinnen und Ärzte in Weiterbildung als (eher) selbständig ein.

Die Resultate dieser Umfrage verdeutlichen, dass die universitäre Ausbildung bei allen medizinischen Fakultäten eine gute Vorbereitung auf den Berufsalltag bietet, aber auch weiterhin Verbesserungspotenzial besteht. Der vermehrte Einsatz von Praktika während des Studiums sowie der Einsatz unterschiedlicher Lehrmethoden könnten dabei zu Verbesserungen führen. Die konsequente Einführung der Kompetenzorientierung in der ärztlichen Ausbildung könnte einen wichtigen Beitrag für die noch bessere Vorbereitung der Medizinstudierenden auf ihre zukünftige Rolle als Ärztinnen und Ärzte leisten. Im Sinne eines Kontinuums zwischen Aus- und Weiterbildung ist insbesondere der direkte Austausch der Beteiligten und Entscheidungsträger im Bereich ärztliche Aus- und Weiterbildung von grosser Wichtigkeit.

\section{Literatur}

1 Sohrmann M, Berendonk C, Nendaz M, Bonvin R. Nationwide introduction of a new competency framework for undergraduate medical curricula: a collaborative approach. Swiss Medical Weekly. 2020;150:w20201.

2 Michaud P, Jucker-Kupper P. Profiles working group. Nendaz M, Bonvin R. The "Profiles" document: a modern revision of the objectives of undergraduate medical studies in Switzerland. Swiss Medical Weekly. 2016;146:w14270.

3 Bearth A, Burgermeister LC, Bauer W, Suetterlin B, Siegrist M. Personalisierte Medizin: Umfrageresultate 2018. Schweiz Ärzteztg. 2019;100(38):1256-9.

4 Van der Horst K, Siegrist M, Orlow P, Berendonk C, Giger M. Demographie, Beurteilung des Studiums und der Feedbackkultur an den Weiterbildungsstätten. Schweiz Ärzteztg. 2010;91(6):203-7. 